\title{
Uso del Catálogo en Bibliotecas de la UNAM
}

Filiberto F. Martínez A.

Investigador del CUIB

\section{RESUMEN}

En estees tu dio se pre sen tan los re sul ta dos ob te ni dos de la apli ca ción de una en cues ta a 749 u sua rios de las bi bliotecas correspondientes a las dife ren tes es cue las y facul ta des de la UNAM. El prin ci pal ob je ti vo de esta en cues ta fue obtener un conjunto de datos generales acerca del uso que se hará del catálogo en las bibliotecas universitarias de esta institución. Algunos de los datos incluidos en este estudio son:

Facultad y semestre que cursan los usuarios de la biblioteca.

Frecuencia de asistencia a la biblioteca.

Mecanismos usados para localizar los materiales bibliográficos.

Punto de acceso empleados en el catálogo.

Datos sobre éxitos y fracasos en la búsqueda de los materiales bibliográficos.

Facilidad o dificultad en el uso del catálogo.

Instrucción recibida por el usuario sobre el uso de la biblioteca.

Asimismo, se presentan algunos cruzamientos entre las diferentes variables incluidas en la encuesta.

Algunos de los resultados a que se llegaron después de analizar los datos son:

El usuario de la biblioteca utiliza el catálogo de la biblioteca únicamente en la mitad de los casos.

Solamente $48.7 \%$ de los usuarios han recibido instrucción sobre el uso de la biblioteca.

Los pun tos de ac ce so del ca tá lo go son usa dos in co rrec ta men te, ya que el usua rio bus ca ma te ria les so bre de ter mi na do tema por au tor o título.

La participación de los bibliotecarios en la instrucción sobre la biblioteca es mínima.

Otros aspectos sobre el uso del catálogo son incluidos de una manera más amplia en este estudio.

\section{ABSTRACT}

This study re ports the re sults ob tai ned in a sur vey applied to 749 users of the li bra ries corresponding to the different schools and fa cul ties of the UNAM. The main ob je ti ve of the sur vey was to ob tain a set of ge ne ral data on the u se of the card ca ta $\log$ in the uni ver sity li bra ries of this ins ti tu tion. Some of the data in clu ded in this study are:

Faculty and semester that library users have attained

Frecuency of library attendance

Mechanisms used to locate bibliographical materials in the library

Access points used in the catalog

Data on successes and failures in the search for bibliographical materials

Simplicity or difficulty in the use of the catalog

Instruction received by the user on the use of the library

Likewise, there are some interrelations between the different variables included in the survey.

Some of the results derived from the analysis of the data are:

The library user utilized the library catalog only in half of the cases

Only $48.7 \%$ of the users have received instruction on the use of the library

The ac cess points to the ca ta $\log$ were used im pro per ly, inas much as the user sear ched ma terials on a de ter mi ned sub ject by author or title

The participation of librarians in the instruction on the library is minimal 
Other aspects on the use of the catalog are included in a more ample manner in this study.

\section{INTRODUCCION}

Todos es ta mos de acuer do en que el ca tá lo go de la bi blio te ca es un elemento de primordial importancia para el usuario de la biblioteca, por lo que una gran mayoría de personas coinciden en califi car lo como "el corazón de labiblio te ca" o como "la lla ve de acceso a la colección".

Dada la importancia que reviste este instrumento, muchos bibliotecarioshemosdestinadounacantidadde esfuerzosconsidera bles a la rea li za ción de ca tá lo gos úti les para el usua rio, sin embar go, al rea li zar el tra ba jo de la ela bo ra ción de es tos ca tálo gos, "fre cuen te men te asu mi mos que el pú bli co habla nues tro mis mo len gua je, o que sus ne ce si da des son aque llas que no so tros per cibi mos a tra vés de nues tra po si ción de gran au to ri dad o que to das las per sonas tie nen la mis ma mi nu cio si dad y so fis ti cación en las búsquedas que nosotros hacemos". (1)

Al asumir esta actitud, podemos producir catálogos que desde nuestro punto de vista sean útiles para nuestros usuarios, pero que en la rea li dad no res pon den a las ex pec ta ti vas de és tos, por lo que es ne ce sario, ade más de or ga ni zar ade cua da men te los ma teriales a través de su catalogaciónpara que el usuario conozca lo que tiene la biblioteca, efectuar estudios que los evalúen, con la finali dad de saber si los ca tá lo gos que es ta mos ela bo ran do sa tisfacen sus ne ce si da des. Por lo an tes ex pues to, se con si de ró per tinente realizar esta investigaciónentre los alumnos que asisten a las bi blio te cas de la UNAM, con la fi nali dad de te ner un pa no rama sobre el uso y utilidad de los catálogos.

\section{ESTUDIOS SOBRE EVALUACION DEL CATALOGO.}

Al revisar la literatura escrita sobre la evaluación del catálogo, podemos darnos cuenta de que existe un sinnúmero de reportes sobre diversos estudios que se han realizado, principalmenteen las bibliotecas norteamericanas.

De toda esta se rie de es tu dios, M.J. Ba tes (2) men cio na al gu nos de ellos agrupados de la siguiente manera:

\section{Estudios sobre cantidades globales de uso del catálogo.}

Son aquellos cuyo objetivo es conocer el volumen total de uso del catálogo, es decir, estudios cuantitativos, entre los que se pue de men cio nar par te del es tu dio lle va do a cabo por Li petz (3) en el catálogo de la Biblioteca de la Universidad de Yale.

2. Estudios sobre la comparación de las búsquedas por autor/título contra búsqueda por materia.

Par ten del he cho de que los usua rios de una bi blio te ca bus can en el ca tá lo go un ma te rial co no ci do, en cuyocaso uti li zan el acce so por au tor o títu lo, obien una se rie de ma te ria les so bre de ter minado tema, em plean do el ac ce so por ma te ria. Los re sul ta dos de estos es tu dios nos mues tran que el uso de los dos ti pos de ac ce so es si mi lar, pues en tre un 20 y $50 \%$ de los usua rios bus can por ma teria y el res to lo ha cen por au tor o tí tu lo. Como ejem plos de los estudios de este tipo se pueden señalar los citados por Montague
(4), así como los de la ALA (5), el de Lipetz (3), y el de Tagliacozzo-Kochen. (6)

\section{Es tu dios so bre la re la ción en tre el uso de ca tá lo go por ma- teria y la experiencia en la materia.}

Sostienen el principio compartido por varios autores sobre la evi den cia de que a me di da que el usua rio tie ne un ni vel aca dé mico más ele va do, el uso del ca tá lo go por ma te ria es me nor en re lación al uso de los autores y títulos. Algunos estudios relacionados con la cuestión anterior son discutidos por Frarey (7) y Montague. (4)

\section{Es tu dios so bre los pro pó si tos para usar el ca tá lo go de ma- teria.}

Estoses tudios mencionan que elca tálogode materiaesutiliza do equitativamente para dos fines: a) Seleccionar libros sobre una materia y b) encontrar la ubicación de los librosen laestan te ría, para efectuar una selección de libros en la misma. Frarey (7) menciona tres estudios de este tipo, los cuales indica, necesitan revisarse bajo condiciones modernas.

\section{Estudios sobre el número de búsquedas en el catálogo de materia.}

Son los que nos proporcionan datos sobre el porcentaje de búsque das úni cas en re la ción con las bús que das múl ti ples al uti lizar el catálogo de materia, es decir, cuántas búsquedas realiza el usuario en este catálogo.

Estos estudios han indicado que entre un 66 y un $77 \%$ de los usuarios buscan únicamente una sola vez en el catálogo de temas. Entre los estudios de este tipo se encuentran el de Knapp (8), el de la ALA (5) y el de Tagliacozzo-Kochen. (6)

\section{Es tu dios so bre la apre cia ción de la efec ti vidad del ca tálogo de materia.}

Este tipo de es tu dios tie nen como ob je ti vo va lo rar la efec ti vi dad del catálogo de materia, lo cual varía considerablemente en los di ver sos es tu dios rea li za dos, de bi do, tal vez, a la va rie dad de criterios utilizados en cada uno de éstos.

Los estudios que señalan un alto índice de efectividad son los mencionados por Fray (7), el de ALA (5) y Lipetz (3). Por otro lado los que reportan un índice de efectividad bajo son los de Knapp (8), Malcom (9) y Quigley. (10)

Por otra parte, Lancaster (11) también cita algunos de los estudios se ña la dos por M.J. Ba tes y al gu nos más como el es tu dio sobre el uso del ca tá lo go en el Rei no Uni do (12), y el es tu dio de requerimientos para futuros catálogos realizado en la Graduate Library School de la Universidad de Chicago. (13)

Para Lancaster, los estudios que se han realizado acerca del uso del ca tá lo go pue den ubi car se en dos gran des gru pos: los es tu dios de macroevaluación y los estudios de microevaluación. 
Los Estudios de macroevaluación arrojan únicamente datos generales sobre el uso del catálogo y no se enfocan a ejemplos específicos de uso del catálogo, mientras que los estudios de microe va lua ción pre ten den co no cer si el usua rio en cuen tra o no los materiales que está buscando, es decir, establecen una diferenciación entre los éxitos y fracasos al utilizar el catálogo, así como las causas que inciden en éstos.

\section{EVALUACION DE CATALOGOS EN MEXICO.}

En el país no se han realizado estudios de este tipo, pues únicamen te exis ten dos do cu men tos que abor dan el asun to, uno ela borado por Estela Morales Campos (14) y una tesis profesional presentada por Isabel Espinosa. (15)

Estela Morales Campos efectuó en 1978, a raíz de la adopción del capítu lo VIde las Re glas de Ca ta lo ga ción An gloame ricanas por la Di rec ción Ge ne ral de Bi blio te cas de la UNAM, un es tu dio tendiente al conocimiento sobre la utilidad para los usuarios de cada uno de los datos incluidos en los registros catalográficos.

A tra vés de la apli ca ción de un cues tio na rio se in da gó la uti li dad de los si guien tes da tos: coau to res, títu los, nú me ro de edi ción, lugar de publicación, editorial, año de publicación, páginas, tipo de ilus tra ciones, ta ma ño, nota de se rie, no tas, tipo de pa gi nación, forma de contenido, tipo de publicación, tipo de reproducción, material complementario e índice analítico.

El es tu dio arro jó una se rie de da tos in te re san tes so bre la uti li dad de cada uno de estos elementos, además de encontrar entre los usuarios un desconocimiento y falta de familiaridad con estos datos.

La tesis: "Uso del catálogo de la Biblioteca Central de la UNAM", presenta los resultados de la apli ca ción de un cues tiona rio a 1.000 usua rios de esta bi blio te ca, to dos ellos apli ca dos a estudiantes de nivel licenciatura. Este estudio nos ofrece datos so bre las ca rac te rís ti cas de los usua rios que asis ten a la bi blio teca en cues tión, ta les como se mes tre, ca rre ra, si han o no re ci bi do orien ta ción so bre el uso del ca tá lo go, opi nión so bre su arre glo y tipo de ma te rial que se bus ca en él, ra zo nes de bús que da en el catálogo, aspectos relacionados con las tarjetas de véase y véase también, así como los resultados obtenidos al efectuar diversas búsquedas con distintos tipos de autores y temas.

$\mathrm{Al}$ ana li zar este es tu dio, se pue de ob ser var que pre ten dió ser un es tu dio muy am plio, el cual tra tó de in ves ti gar una gran can ti dad de cuestiones, muchas de las cuales pueden resultar motivo de in ves ti ga ción por sí so las. Al tra tar de abar car una se rie de cuestiones muy amplias, no llega a conclusiones muy claras acerca del com por ta mien to de los usua rios del ca tálogo de la Biblio te ca Cen tral de la UNAM, sin em bar go, la se rie de da tos que se re copilaron son de gran utilidad, pues re pre sen tan un pri mer acerca- miento hacia el conocimiento de la gente que utiliza los catálogos en nuestras bibliotecas.

\section{OBJETIVO DE ESTUDIO}

El obje ti vo pri mor dial de este es tu dio fue ob te ner un con jun to de da tos ge ne ra les acerca de los usua rios de las di ver sas bi blio te cas de la UNAM, así como del uso que hacen del catálogo, y aquellos fac to res que in flu yen po si ti va o ne ga ti va men te en di cho uso, tales como la educación en el uso del catálogo. Con base en lo an tes ex pues to, pue de con si de rar se que la par te cen tral del es tudio giró en torno al conocimiento de las necesidades que llevan al usua rio a vi si tar la bi blio te ca, es de cir, si su vi si ta es mo ti va da por la consulta de una obra en particular o varias sobre un tema de ter minado. Asi mis mo, se in da gó so bre una se rie de cues tiones adicionales, las cuales pudiesen haber influido dentro de los resul ta dos ob te nidos al localizarel mate rial de sea do. Den trode las cuestiones adicionales investigadas se pueden mencionar: meca nis mos de ac ce soutiliza dos al ma ne jarel catálogo, fre cuencia de asis ten cia a la bi blio te ca, se mes tre cur sa do y una se rie de puntos en re la ción con la ins truc ción re ci bi da para el uso del ca tálogo.

\section{METODOLOGIA}

Si con si de ra mos que el es tu dio te nía como ob je ti vo prin ci pal recabar da tos ge ne ra les so bre las per so nas que uti lizan el ca tálo go en las bi blio te cas de la UNAM, y so bre al gu nos fac to res que influyeron en los resultados obtenidos, se consideró pertinente la uti liza ción de un cues tio na rio, en lu gar de la ob serva ción di rec ta del uso que se hace del catálogo.

Se tomó como mo de lo el cues tio na rio uti liza do en el es tu dio sobre el uso del ca tá lo go en el Rei no Uni do (12), al que se le hi cieron al gu nas mo di fica cio nes y el cual es pre sen ta do como ane xo. El apli car di cho cues tio na rio como mo de lo, obe de ció a que el estu dio lle va do a cabo en las bi blio te cas de la UNAM, al igual que el realizado en las bibliotecas del Reino Unido, tuvieron como objetivo lle var a cabo un es tu dio cuan ti ta ti vo más que cua li ta tivo, es decir, un estudio de macroevaluación.

En el caso de la UNAM, de bi do a las di fe ren tes ca rac te rís ti cas de los ca tá lo gos de las di ver sas bi blio te cas, así como de la co mu nidad de cada una de las es cue las y fa cul ta des, es muy di fícil rea lizar un estudio general de microevaluación, donde se analicen éxi tos y fra ca sos al bus car in for ma ción en los ca tá lo gos, ade más de pre cisare iden ti ficar las po sibles cau sas. Este tipo de es tu dios de microe valuación es po sible y con ve nien te rea lizar los en el ca tálogo de cada una de las escuelas y facultades.

El es tu dio fue rea li za do me dian te la apli ca ción de un cues tio nario a 749 usua rios de las bi blio te cas de las di ver sas es cue las y facultades de la UNAM. Durante el período comprendido del 10. de no viem bre al 15 de di ciem bre de 1987 se apli ca ron los cuestionarios correspondientes a 18 planteles y del 18 de abril al 31 de mayo, a 4 planteles faltantes en el primer período. 
Para la realización del trabajo de aplicación del cuestionario se con tó con el apo yo de los alum nos del ter cer se mes tre de la li cencia tu ra en bi blio te co lo gía 1987, quie nes a tra vés de en tre vis tas a usua rios to ma dos al azar, re ca ba ron la in for ma ción so li ci ta da en los cuestionarios.

El nú me ro de alum nos que se pre ten dió en cues tar en cada una de las escuelas o facultades fue de 40, no lográndose esto en algunos casos. En el cuadro número uno puede observarse la cantidad de alum nos en cues ta dos en cada uno de los di ver sos plan teles.

Por otro lado, con la finalidad de podercompararlos resultados ob te ni dos en tre los alum nos de los pri me ros se mes tres con los de los últimos, se procuró que las cantidades entre ambos sectores fue ran si mi la res, en cues tan do a 327 alum nos (43.6\%) que cur saban del primero al cuarto se mes tre y a 403 alum nos (53.8\%) del quinto se mes tre en ade lan te, en este úl ti mo gru po fue ron in cluidos algunos pasantes, así como alumnos de posgrado, un ligero nú me ro de alum nos 19 (2.6\%) no in di ca ron el se mes tre que cursaban.

El nú me ro de alum nos per te ne cien te a cada uno de los se mes tres pueden ser observados en el cuadro número dos.

\section{RESULTADOS OBTENIDOS}

\section{a) Principal motivo de asistencia a la biblioteca}

Como se men cio nó an te rior men te, la cues tión cen tral de este estu dio fue in ves ti gar cuál era el prin ci pal mo ti vo de asis ten cia a la biblioteca, si consultar una obra en particular o consultar varias obras so bre un tema, al cues tio nar este pun to se in clu yó una terce ra op ción que fue otras ra zo nes, de bi do a que en oca sio nes algu nos de los usua rios de nues tras bi blio te cas acu den a ellas únicamente a realizar tareas o estudiar en sus propios apuntes.

En el Cuadro 3, se presentan los resultados en relación con este aspecto y podemos observar que los 749 alumnos dieron 810 respuestas, en virtud de que se podía elegir más de una opción. La distribución de las respuestas es la siguiente: 169 (20.8\%) con tes ta ron que acu den a la bi blio te ca a bus car una obra en par ticular, mientras que $503(62.1 \%)$ acuden a buscar más obras sobre un tema, y solamente $138(17.1 \%)$ por otras razones.

Al analizar los resultados anteriores, podemos observar que la principal ra zón por la cual acu den los alum nos a la bi blio te ca es consultar varias obras sobre un tema.

Lo anterior no coincide con lo mencionado por M.J. Bates (2), cuando señala que los estudios realizados sobre la evaluación del catálogo han encontrado que las búsquedas efectuadas por au tor/títu loson si mi la res a las rea li za das por ma teria, pues to que los re sul ta dos que nos arro ja esta en cues ta, exis te una di fe ren cia notable en tre es tos dos ti pos de bús que da. Pues mien tras $62.1 \%$ de nuestros lectores acuden a buscar materiales sobre un tema, solamente $20.8 \%$ lo hacen para tratar de localizar una obra en particular.
Por otra par te, en tre los au to res que han abor da do este asun to, se maneja como un principio que a me di da que el ni vel aca dé mi co del usua rio es más alto, la ne ce si dad de ma te ria les so bre un tema dis mi nu ye, por lo cual se de ci dió com pa rar las res pues tas de los alum nos de pri me ro a cuar to se mes tre con las de los alum nos de se mes tres pos te riores, en con tran do los resul ta dos in clui dos en el cuadro tres y que a continuación se mencionan:

La búsqueda de varios materiales sobre una materia es similar en tre los dos gru pos: en el caso de los alum nos de pri me ro a cuarto semestre $60.5 \%$, en el caso de los alumnos de los semestres pos te rio res li ge ra men te ma yor $63.8 \%$. En los ca sos don de el objetivo de acu dir a la bi blio te ca fue con sul tar una obra en par ti cular, entre los alumnos, de primero a cuarto, el porcentaje fue de $24.9 \%$, mientras que en los de quinto semestre en adelante disminuyó a $18.0 \%$.

Con la fi na li dad de com pro bar real men te que el usua rio acu día a la bi blio te ca a bus car una obra en par ti cu lar o va rias obras so bre un tema, se le pi dió men cio na ra den tro del cues tio na rio el au tor o título de la obra en cuestión, o el tema de las obras deseadas.

En el Cua dro 4, se pre sen tan los da tos acer ca de los usua rios que incluyeron estos datos, encontrando que un $85.7 \%$ los proporcionaron.

Con los resultados anteriormente expuestos, podemos afirmar que en relidad los alumnos encuestados buscaban una obra en particular o varias obras sobre un tema, y que por lo tanto, el prin ci pio que sos tie ne la dis mi nu ción de la bús que da por ma te ria y el au men to de la de au tor/títu lo, a me di da que el ni vel aca dé mico es más alto, no es váli do den tro de los in te gran tes de un mismo ni vel, en este caso es tu dian tes de ni vel li cen cia tu ra. Qui zá si comparásemos estudiantes de licenciatura con estudiantes de posgrado los resultados serían otros.

Otro as pec to que se in ves ti gó fue co no cer qué me ca nis mos uti liza el usua rio para lo ca li zar las obras de sea das, plan teán do se cua tro alternativas: efectuar una búsqueda en el catálogo de la biblioteca, preguntar al bibliotecario, buscar directamente en la estantería, o algún otro mecanismo.

Los re sul ta dos ob te ni dos in clui dos en el Cua dro 5 , fue ron los siguien tes: el 53.4\% uti li za el ca tá lo go de la bi blio te ca, se gui do de un $23.8 \%$ que se dirige a buscar en la estantería, los que consultan al bibliotecario representan un $17.4 \%$, y aquéllos que emplean otro mecanismo fueron solamente $5.4 \%$.

Como puede observarse, de los mecanismos utilizados para tratar de lo ca li zar en la bi blio te ca el ma te rial de sea do, el uso del catálogo se emplea únicamente en aproximadamente la mitad de los casos $(53.4 \%)$.

Lo anterior puede tener su causa en que únicamente un número reducido de alumnos han recibido instrucción sobre cómo usar el catálogo. De los 643 alum nos cuyo ob je ti vo de vi si tar a la biblio te ca fue con sul tar una obra en par ti cu lar o va rias obras so bre un tema, sólo 281 alumnos $(43.7 \%)$ declararon haber recibido instrucción de usuarios, de acuerdo con los datos del cuadro diez. 
Otro as pec to im por tan te de in ves ti gar fue el pun to de ac ce so más em plea do al ha cer uso de los ca tálo gos ob te nien do los re sul ta dos siguientes:

Si par ti mos del he cho enun cia do lí neas atrás, de que 503 ve ces $(62.1 \%)$ el motivo de la visita a la biblioteca fue consultar varias obras sobre un tema, y solamente en 169 ocasiones (20.8\%) se acudió a consultar una obra específica, es lógico pen sar que el pun to de ac ce so más uti li za do haya sido el de materia, pero los resultados de la encuesta son totalmente contradictorios, pues si observamos los datos del cuadro seis, podemos darnos cuenta que el acceso por materia es el menos utilizado, pues se recurre a éste solamente en el $23.4 \%$ de los ca sos. En tre los alum nos que bus can una obra en par ti cu lar, los puntos de acceso por autor/título son los más utilizados, pero acon te ce lo mis mo con los alum nos que de sean va rias obras sobre un tema, en este úl ti mo caso uti li zan el ac ce so por au tor en un $39.5 \%$, y el de tí tu lo en un $34.8 \%$ de las oca sio nes, con tra solamente un $25.7 \%$ el de materia.

Lo anterior puede tener una explicación en el bajo índice de usua rios que han re ci bi do ins truc ción so bre el uso del ca tá lo go, y que quizá muchos de los que aseveraron asistir a la biblioteca a con sul tar va rias obras so bre un tema, en rea li dad bus ca ban al gunas obras co no ci das para la rea li za ción de un tra ba jo en mar ca do de una materia. Sería conveniente a futuro realizar otra investigación que ahonde sobre este asunto.

\section{b) Resultados obtenidos al tratar de localizar el material y factores que influyeron:}

Los casos en los cuales se visitó a la biblioteca para consultar obras fueron 672: para tratar de localizar una obra en particular 169 ocasiones y varias obras sobre un tema 503 veces. En un $69.3 \%$ de los ca sos se en con tró el ma te rial que se bus ca ba, ob teniendo un éxito ligeramente mayor los alumnos que buscaron una obra en par ti cu lar $(71.0 \%)$, en re la ción con los que qui sie ron consultar varias obras sobre un tema $(68.7 \%)$.

Al analizar algunos de los cuadros que se presentan dentro de este reporte, podemos observar los siguientes datos:

En el Cuadro 8, tenemos que 426 alumnos (66.2\%) acuden a la bi blio te ca más de una vez a la se ma na, 158 alum nos (24.6\%) semanalmente, y 59 (9.2\%) una vez al mes. Sería lógico suponer que los alumnos que más frecuentemente asisten a la biblioteca son los que en cuen tran una can ti dad ma yor de ma te rial, sin embargo los que tienen una frecuencia de asistencia mensual o semanal tienen un éxito ligeramente mayor, puesto que los que asis ten men sual men te en cuen tran el ma te rial en e171.2\% de los casos y los que asisten semanalmente encuentran el material en un $70.3 \%$ y los que asisten más de una vez a la semana únicamente en un $66.2 \%$ de las veces.

Puede ser, aunque habría que comprobarlo, que las necesidades de aque llos alum nos con ma yor fre cuen cia de asis ten cia a la biblioteca sean mayores, en relación con las de los alumnos con una asistencia esporádica.
Otro de los fac to res, de los cua les tra tó de ob ser var se su in fluencia en la localización del material deseado, fue el semestre que cursaban los alumnos, obteniendo los resulta dos in cluidos en el cuadro nueve y mencionados en seguida:

Dado que los alum nos del quin to se mes tre en ade lan te han te nido mayor opor tu ni dad de asis tir a la bi blio te ca, po dría pen sar se que debido a la experiencia ad qui ri da, este gru po ten dría el porcentaje más alto de éxito al tratar de localizar los materia les deseados, sin embargo, acontece lo contrario, mientras que los alum nos de pri me ro a cuar to se mes tre en cuen tran los ma te ria les en un $73.8 \%$, los del quin to se mes tre en ade lan te lo lo gran so lamente en el $65.1 \%$ de los casos.

En re la ción a lo an te rior, cabe ha cer los si guien tes co men ta rios: ¿nue stras bi blio te cas úni ca men te es tán aten dien do las ne ce si dades de los alum nos de los prime ros se mes tres? ¿las ne ce si da des de información bibliográfica de los alumnos de los últimos semes tres son ma yo res a los de los pri me ros se mes tres? o las condiciones económicas por las que atravesamos ha hecho que los alum nos que cur san ac tual men te los pri me ros se mes tres acu dan con ma yor fre cuen cia a la bi blio te ca y por lo tan to ten gan me jor experiencia en localizar los materiales.

Estas interrogantes son cuestiones que se deben investigar para en con trar las po si bles cau sas y ex pli ca ción a este fe nó me no, asî como el planteamiento de las soluciones adecuadas.

Por úl ti mo, se tra tó de in da gar si el ha ber re ci bi do ins truc ción sobre el uso del ca tá lo go in flu yó para te neréxi to en la lo ca li za ción de los ma te ria les, en con tran do que el por cen taje de alum nos que encuentran el material es similar tanto en los que han recibido cursos de instrucción de uso del ca tálo go, como en aqué llos que no lo han re ci bi do. Los que re ci bie ron cur sos en con tra ron el mate rial en un $69.4 \%$ de los ca sos, mien tras que los que no los re cibieron localizaron en $68.8 \%$ de los casos

Lo an te rior pue de te ner ex plica ción, al re cor dar que úni ca mente cer ca de la mi tad de los ca sos $(53.4 \%)$ lo uti li za como me canismo para localizar las obras en la biblioteca la consulta del catálogo.

\section{c) Instrucción sobre el uso del catálogo:}

Teniendo en cuenta que la instrucción sobre el uso del catálogo es uno de los elementos más importantes, para hacer de éstos, instrumentos útiles al servicio de los usuarios, se incluyeron en la encuesta una serie de cuestiones tendientes a conocer quién im par te ésta y cómo la con si de ran los asis ten tes a la bi blio te ca.

Al analizar los datos incluidos en el Cuadro 10, percibimos que de los 294 alum nos que cur sa ban del pri me ro al cuar to se mes tre únicamente 131 (44.5\%) habíanrecibido algunainstrucción sobre el uso del ca tá lo go, por cen ta je que es si mi lar en tre los alumnos de los últimos semestres, pues en este grupo 144 recibieron instrucción (43.2\%). 
Lo an te rior hace su po ner que la ins truc ción so bre el uso del ca tálogo se da en los primeros semestres, no volviéndose a ofrecer entre los alumnos de los últimos.

Al indagar entre los usuarios respecto a la facilidad para usar el catálogo, se encontró que aquéllos que han recibido alguna instrucción, les parece fácil en un $85.8 \%$, y en cam bio a los que no la han recibido les parece fácil su uso solamente en un $73.2 \%$.

Aquí podemos decir que no existe ningún problema, pues aunque una gran ma yo ría no ha re ci bi do cur sos de ins truc ción se les facilita el uso del catálogo, pero debemos tener cuidado, pues muchas veces los usuarios parecen estar satisfechos porque no co no cen las po si bi li da des del ca tá lo go y no de sean algo más allá de sus expectativas.

Por último, al investigar entre los alumnos que habían recibido instrucciones sobre el uso del ca tá lo go, 195 de ellos (69.3\%) indi ca ron que ésta ha bía sido su fi cien te, y 86 alum nos (30.7\%) señalaron que la consideraban insuficiente, por otro lado, los bibliotecarios participaron directamente en esta instrucción so la men te en 77 ca sos $(21.7 \%)$, mien tras que de acuer do con los da tos del cua dro tre ce, los maes tros lo hi cie ron 109 ve ces, es decir, en un $(30.8 \%)$.

\section{CONCLUSIONES}

Se han presentado los datos más relevantes sobre esta encuesta lle va da a cabo en tre los alum nos que asis ten a las di ver sas bi blio- tecas de las escuelas y facultades de la UNAM, sin embargo, analizando los cuadros que se presentan al final de este trabajo, pue den in fe rir se otra se rie de da tos ob te ni dos a tra vés de la rea lización de este estudio, los cuales no han sido expuestos por haber deseado exponer únicamente los más relevantes.

Al ha cer un re su men de lo ex pues to en este ar tícu lo po de mos llegar a las siguientes conclusiones:

Siempre hemos partido del hecho de que el catálogo es de gran uti li dad para el usua rio, ya que a tra vés de él se pue de sa ber si la biblio te ca tie ne los ma te ria les que el usua rio de sea, sin em bar go ve mos que sólo se hace uso de él en la mi tad de los ca sos. Lo anterior tiene su ori gen en la poca ins truc ción que se le da al usuario sobre el uso del catálogo, ya que solamente un $48.7 \%$ de los alumnos encuestados han recibido este tipo de instrucción.

Esta fal ta de ins truc ción hace que exis tan si tua cio nes, las cua les pa re cen to tal men te iló gi cas, como en el caso de que los usua rios bus can por au tor y títu lo, cuan do su ma yor ne ce si dad es con sultar va rias obras so bre un tema, lo que es ex pli ca ble, por que nunca se les ha indicado qué es un tema y qué tipo de material pueden encontrar bajo este punto de acceso.

Lain ter ven ción del bi blio te ca rio en los cur sos so bre ins truc ción de usua rios ha sido míni ma, y si que re mos que efec ti va men te el ca tá lo go de la bi blio te ca se con vier ta en un ins tru men to a tra vés del cual el usua rio pue da te ner ac ce so a la co lec ción, nues tra participación en los cursos de instrucción sobre su uso debe incrementarse, pues de lo contrario seguiremos elaborandocatálogos muy bien he chos des de nues tro pun to de vis ta, pero los cua les no se utilizarán adecuadamente.

\section{REFERENCIAS}

1. DWYER, James R. “Public responsetoanacademiclibrarymicrocatalog”. — Journal of Academic Librarianship. - v. 5, no. 3 (jul. 1979). - p. 136.

2. BATES, Marcia J. "Factors affecting subject catalog search success". - Journal of the American Society for Information Science. - v. 28 (may 1977) p. 162.

3. LIPETZ, B.A. User require ments in iden tifying de siredworks in alarge library. New Haven, Ct: Yale University library, 1970. citado por M.J. Bates.

4. MONTAGUE, E. A. Card catalogue use studies 1949-1965. - M. A. Thesis, University of Chicago, 1967. citado por M.J. Bates.

5. AMERICAN LIBRARY ASSOCIATION. Resources and Technical Services. Cataloging and Classification Section. Catalogue use study -- Chicago : ALA, 1958. citado por M.J. Bates.

6. TA GLIA COZZO, R; Ko chen, M. Information-seeking behavior of ca ta lo gue users". - In for mation Storage and Retrieval. - v. 6, no. 5 (dic. 1970) citado por M.J. Bates. 
7. FRA REY, C. J. Studies of use of the subjectca ta log: summary and eva lua tion. En: The sub ject analy sis of library materials. Maurice F. Tauber. - New York : Columbia University, School of Library Service, 1953. - p. 147 - 166. citado por M.J. Bates.

8. KNAPP, P. B. “The subjectcatalog in the Colle gelibrary: an inves tigation ofterminology". - The Library Quarterly. - v. 12, no. 3 (jul. 1944). citado por M.J. Bates.

9. MAL COM, R. S. “The stu dent's approach to the card ca ta log: a study ba sed on a survey of stu dent use at the Library of the Uni ver sity of Pittsburgh". - M.L.S. Te sis. Carne gie. Library School, Carne gie Ins ti tute of Technology, 1950. citado por M.J. Bates.

10. QUI GLEY, H. “An investigation of the pos sible relationship of interbranchloantocataloguing”. - The Library Quaterly. - v. 14, no. 4 (oct. 1944). citado por M.J. Bates.

11. LANCAS TER, F. W. “Estudios sobre uso del ca tálogo”. EnEvalua ción y me di ción delos servi cios bibliotecarios. - México : UNAM. Dirección General de Bibliotecas, 1983. - p. 21 - 83.

12. MALTBY, A ; Sweeney R. “The UK catalogue use survey. - Journal of Librarianship. — v. 4, no. 3 (jul. 1972).

13. UNIVERSITY OF CHICAGO. Graduate Library School "Requirements study for future catalogs". Progress Report. No. 2. Chicago, 1968. citado por F. W. Lancaster.

14. MORALES Campos, Estela "Necesidades de los usuarios". En El Formato MARC II en la automatización de bibliotecas. - México : UNAM. Dirección General de Bibliotecas, 1981. — p. 21 - 27.

15. ESPINOSA Becerril, Isabel "Uso del Catálogo de la Biblioteca Central de la UNAM". - Tesis Licenciado en Bibliotecología. UNAM. Facultad de Filosofía y Letras. - México, 1987.

\section{BIBLIOGRAFIA CONSULTADA}

1. BATES, Marcia J. “Factores affecting subject catalog search success. Journal of the American Society for Information Science. — v. 28 (mayo 1977). — p. 161 - 169.

2. DWYER, James R. "Public responsetoanacademiclibrarymicrocatalog”. — Journal of Academic Librarianship. - v. 5, no. 3 (jul. 1979). - p. 132 - 140.

3. ESPINOSA Becerril, Isabel "Uso del catálogo de la Biblio te ca Cen tralde la UNAM. - Tesis licenciadoen Bibliotecología. UNAM. Facultad de Filosofía y Letras. - México, 1987. - 105 p.

4. FROST, Ca roly O. “Facultyuse of subjectsearching incardandonline catalogs. — Jour nal of Aca de mic Librarianship. - v. 13, no. 2 (mayo 1987). - p. 86 - 92.

5. LANCAS TER, F.W. “Estudios sobre uso del catálogo”. En evaluación y me dición de los servicios biblio tecarios. - México : UNAM. Dirección General de Bibliotecas, 1983. - p. 21 - 83.

6. MALTBY, A ; Sweeney, R. “The UK catalogue use survey”. — Journal of Librarianship. — v, 4, no. 3 (jul. 1972). p. 188 - 204.

7. MORALESCampos, Este la “Necesidadesdelosusuarios”. En elFor ma to MARC II en la au to ma ti za ción de biblioteca. - México : UNAM. Dirección General de Bibliotecas, 1981. — p. 21 - 27.

8. SAWANSON, Don. R. "Requeriments study for future catalogs". - The Library Quaterly. - v. 4 (jul. 1972). - p. 302-315. 
9. TAGLIACOZZO, R. : Ro sen berg, L; Ko che, M. “Acces andrecog nition:from user's data to ca talo gue entries”. - Journal of Documentation. - v. 26, no. 3 (sep. 1970). - p. 230 - 249.

10. LANCAS TER, F.W. “Estudios so bre uso del ca tálo go”. En Evalua ción y me di ción de los servi cios bi blio tecarios. - México : UNAM. Dirección General de Bibliotecas, 1983. - p. 21 - 83.

11. MALTBY, A ; Sweeney R. “The UK catalogue use survey”. - Journal of Librarianship. — v. 4, no. 3 (jul. 1972).

12. UNIVERSITY OF CHICAGO. Graduate Library School "Requeriments study for future catalogs". Progress Report. No. 2. Chicago, 1968. citado por F. W. Lancaster.

13. MORALESCampos, Este la “Necesidadesdelosusuarios" En El For ma to MARCII en la au to ma ti za ción de bibliotecas. - México : UNAM. Dirección General de Bibliotecas, 1981. — p. 21 - 27.

14. ESPINOS A Becerril, Isabel "Uso del Catálogo de la Biblioteca Central de la UNAM". — Tesis Licenciado en Bibliotecología. UNAM. Facultad de Filosofía y Letras. - México, 1987. 
ANEXOS

Cuadro 1

Número de alumnos por facultad o escuela

\begin{tabular}{|l|c|c|}
\hline NOMBRE DE LA FACULTAD O ESCUELA & $\begin{array}{c}\text { No, DE } \\
\text { ALUMNOS }\end{array}$ & $\%$ \\
\hline FACULTAD DE CIENCIAS POLITICAS Y SOCIALES & 42 & 5.61 \\
FACULTAD DE PSICOLOGIA & 53 & 7.08 \\
FACULTAD DE MEDICINA VETERINARIA Y ZOOTECNIA & 40 & 5.34 \\
FACULTAD DE FILOSOFIA Y LETRAS & 40 & 5.34 \\
FACULTAD DE CONTADURIA Y ADMINISTRACION & 19 & 2.54 \\
FACULTAD DE QUIMICA & 42 & 5.61 \\
FACULTAD DE ODONTOLOGIA & 37 & 4.94 \\
FACULTAD DE INGENIERIA & 39 & 5.20 \\
FACULTAD DE ECONOMIA & 29 & 3.87 \\
FACULTAD DE ARQUITEGTURA & 21 & 2.80 \\
FACULTAD DE MEDICINA & 56 & 7.48 \\
FACULTAD DE DERECHO & 37 & 4.94 \\
FACULTAD DE CIENCIAS & 31 & 4.14 \\
ESCUELA NACIONAL DE MUSICA & 40 & 5.3 .4 \\
ESCUELA NACIONAL DE ENFERMERIA Y OBSTETRICIA & 20 & 2.67 \\
ESCUELA NACIONAL DE TRABAJO SOCIAL & 40 & 5.34 \\
ESCUELA NACIONAL DE ARTES PLASTICAS & 19 & 2.54 \\
ESCUELA NACIONAL DE ESTUDIOS PROFESIONALES "IZTACALA" & 14 & 1.87 \\
ESCUELA NACIONAL DE ESTUDIOS PROFESIONALES "ZARAGOZA" & 20 & 2.67 \\
ESCLELA NACIONAL DE ESTUDIOS PROFESIONALES "ACATLAN" & 40 & 5.34 \\
ESCUELA NACIONAL DE ESTUDIOS PROFESIONALES "ARAGON" & 29 & 3.87 \\
FACULTAD DE ESTUDIOS SUPERIORES "CUAUTITLAN" & 41 & 5.47 \\
& 749 & \\
\hline
\end{tabular}


Cuadro 2

Semestre al que pertenecían los alumnos

\begin{tabular}{|l|c|c|}
\hline S E M E S T R E & No. DE ALUMNOS & $\%$ \\
\hline PRIMER SEMESTRE & 117 & 15.6 \\
SEGUNDO SEMESTRE & 27 & 3.6 \\
TERCER SEMESTRE & 142 & 19.0 \\
CUARTO SEMESTRE & $\underline{41}$ & $\mathbf{5 . 4}$ \\
T O T A L : & 327 & 43.6 \\
\hline
\end{tabular}

\begin{tabular}{|l|r|r|}
\hline QUINTO SEMESTRE & 119 & 15.9 \\
SEXTO SEMESTRE & 46 & 6.1 \\
SEPTIMO SEMESTRE & 92 & 12.3 \\
OCTAVO SEMESTRE & 27 & 3.6 \\
NOVENO SEMESTRE & 56 & 7.5 \\
DECIMO SEMESTRE & 18 & 2.4 \\
DECIMO PRIMER SEMESTRE & 3 & 0.4 \\
DECIMO SEGUNDO SEMESTRE & 2 & 0.3 \\
P A S A N T E S & 35 & 4.6 \\
P O S G R A D O & 5 & 0.7 \\
T O T A L : & 403 & 53.8 \\
\hline
\end{tabular}


Cuadro 3

Relación entre el semestre que cursaban los alumnos y su objetivo al acudir a la biblioteca

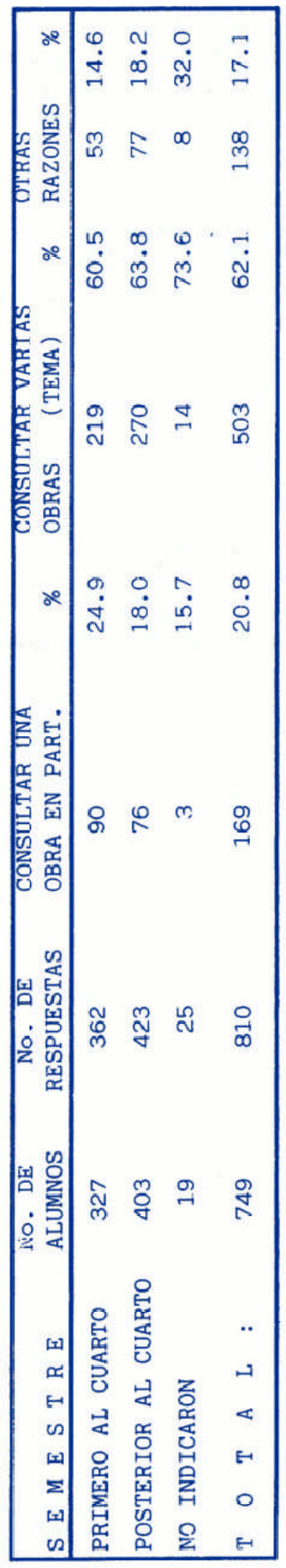

$$
\stackrel{2}{\stackrel{0}{1}} \text { ğ }
$$

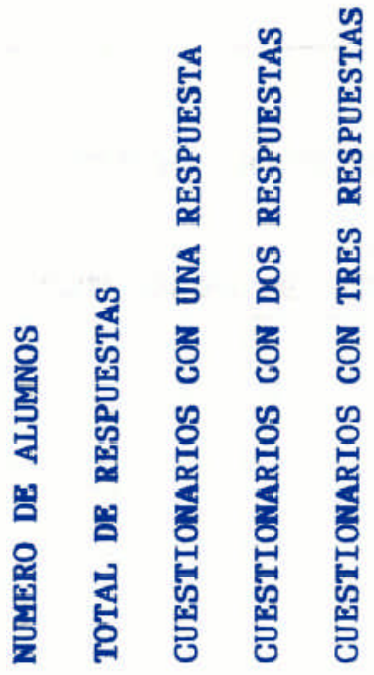


Cuadro 4

Cantidad de alumnos que incluyeron datos sobre el material que buscaban en la biblioteca

\begin{tabular}{|c|c|c|c|c|c|}
\hline \multirow{2}{*}{$\begin{array}{l}\text { OBJETIVO DE VISITA } \\
\text { A LA BIBLIOTECA }\end{array}$} & \multirow{2}{*}{$\begin{array}{c}\text { No. DE } \\
\text { RESPUESTAS }\end{array}$} & \multirow{2}{*}{$\begin{array}{l}\text { INCLUYERON } \\
\text { DATOS }\end{array}$} & \multicolumn{3}{|c|}{ NO INCLUYEN } \\
\hline & & & $\%$ & DATOS & $\%$ \\
\hline CONSULTAR UNA OBRA & & & & & \\
\hline EN PARTICULAR & 169 & 151 & 89.3 & 18 & 10.7 \\
\hline CONSULTAR VARIAS & & & & & \\
\hline OBRAS (TEMAS) & 503 & 425 & 84.5 & 78 & 15.5 \\
\hline 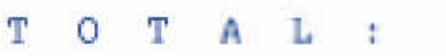 & 672 & 576 & 85.7 & 96 & 14.3 \\
\hline
\end{tabular}

NUMERO DE ALUMNOS

643

TOTAL DE RESPUESTAS

672 
Cuadro 5

Datos sobre mecanismos usados para tratar de localizar

las obras y resultados obtenidos

\begin{tabular}{|c|c|c|c|c|c|c|c|c|}
\hline \multicolumn{4}{|c|}{ MECANISMO UTILIZADO } & $\begin{array}{c}\text { NO. DE } \\
\text { RESPUESTAS }\end{array}$ & $\begin{array}{l}\text { ENCONIFARON } \\
\text { EL MATERIAL }\end{array}$ & $\%$ & $\begin{array}{l}\text { TO BNCOWTKAHON } \\
\text { EL MATEAIAL }\end{array}$ & $\%$ \\
\hline \multirow{2}{*}{\multicolumn{4}{|c|}{$\begin{array}{l}\text { BUSQUEDA EN CATALOGO } \\
\text { DE LA BIBLIOTECA }\end{array}$}} & 453 & 291 & 64.2 & 162 & 35.8 \\
\hline & & & & $(53,4)$ & & & & \\
\hline \multirow{2}{*}{\multicolumn{2}{|c|}{$\begin{array}{l}\text { PREGUNTA } \\
\text { TECARIO }\end{array}$}} & AL & BIBLIO- & 148 & 100 & 67.6 & 48 & 32.4 \\
\hline & & & & $(17.4)$ & & & ti & \\
\hline \multirow{2}{*}{\multicolumn{2}{|c|}{$\begin{array}{l}\text { BUSQUEDA } \\
\text { TERIA }\end{array}$}} & EN & LA ESTAN & 202 & 143 & 70.8 & 59 & 29.2 \\
\hline & & & & $(23.8)$ & & & & \\
\hline 0 & $\mathbf{T} \mathbb{R}$ & 0 & $\mathbf{s}$ & $\begin{array}{c}46 \\
(5.4\}\end{array}$ & 30 & 65.2 & 16 & 34.6 \\
\hline \multirow[t]{2}{*}{$\mathrm{T}$} & $0 \mathrm{~T}$ & A & L : & 849 & 564 & 66.4 & 285 & 33,6 \\
\hline & & & & $(100)$ & & & & \\
\hline
\end{tabular}

NUMERO DE ALUMNOS

643

TOTAL DE RESPUESTAS

849

CUESTIONARIOS CON UNA RESPUESTA

488

CUESTIONARIOS CON DOS RESPUESTAS

114

CUESTIONARIOS CON TRES RESPUESTAS

31

CUESTIONARIOS CON CUATRO RESPUESTAS

10 
Cuadro 6

Datos sobre el punto de acceso empleado en el catálogo por los alumnos que asisten a la biblioteca para consultar materiales

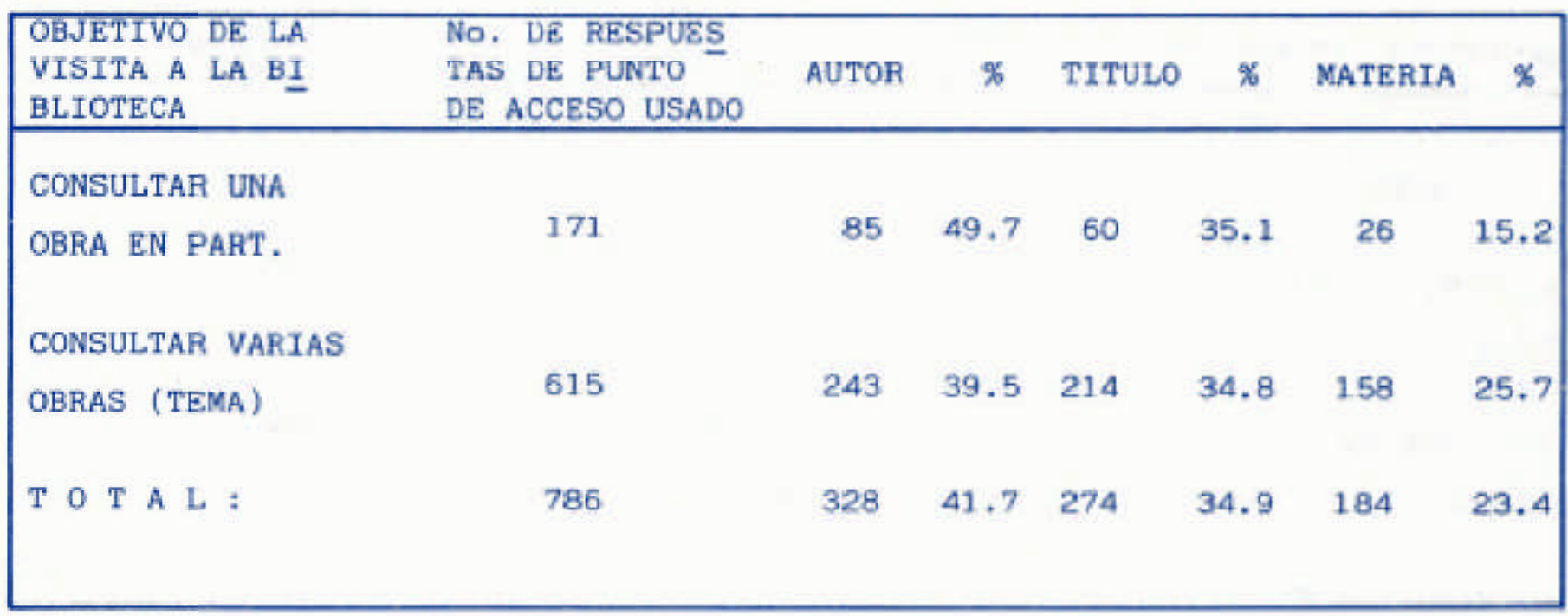

NUMERO DE ALURNOS

TOTAL DE RESPUESTAS 
Cuadro 7

Datos sobre el material encontrado por parte de los alumnos que desean consultar una o varias obras

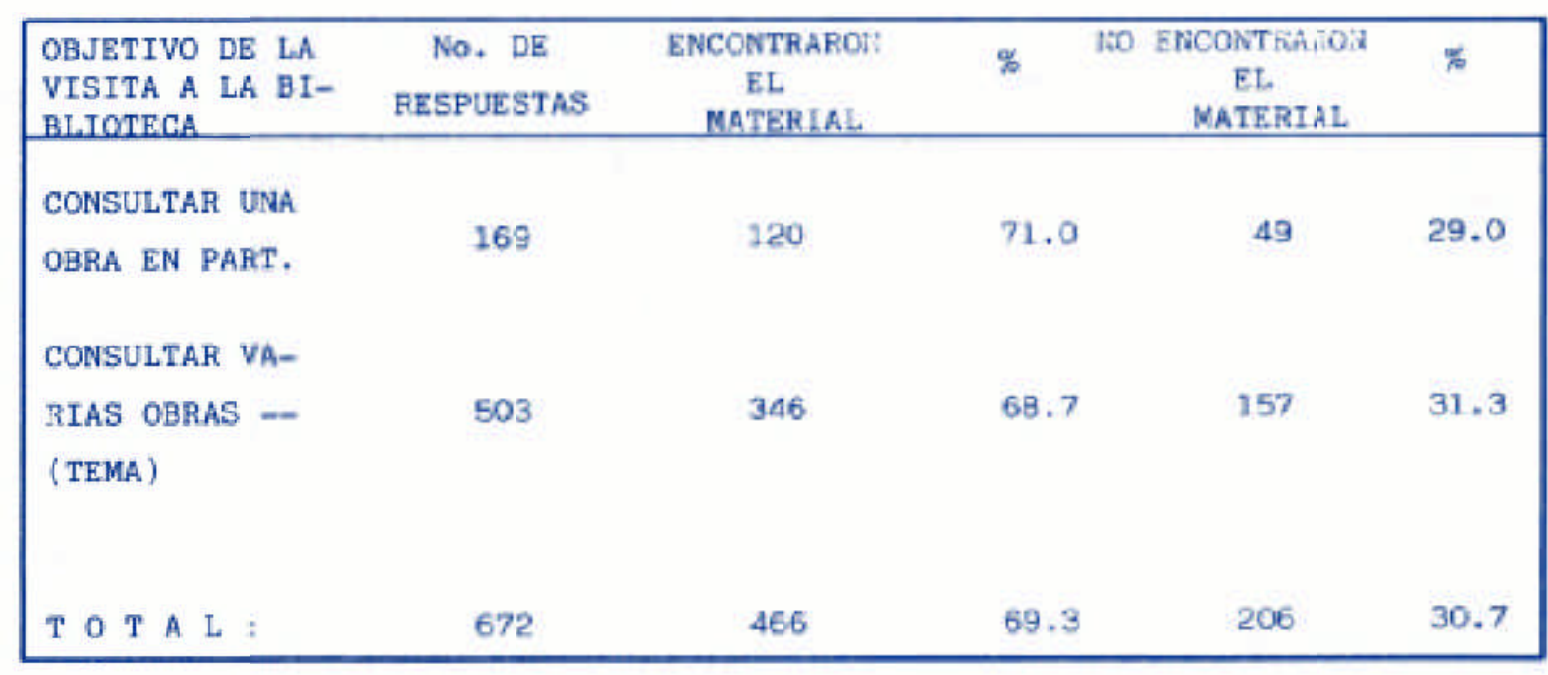

NURERO DE NLIRNOS

643

NURERO DE RESPUESTAS

672

Cuadro 8

Relación entre la frecuencia de asistencia a la biblioteca y el resultado obtenido al localizar el material

\begin{tabular}{|c|c|c|c|c|c|c|}
\hline $\begin{array}{l}\text { FRECUENCIA DE ASIS- } \\
\text { TENCIA A LA BIBLIOTECA }\end{array}$ & $\begin{array}{r}\text { No. DE } \\
\text { ALUMNOS } \\
\end{array}$ & $\begin{array}{l}\text { ENCONTRARON } \\
\text { EL MATERIAL }\end{array}$ & $\%$ & $\begin{array}{l}\text { NO } \\
\text { EL }\end{array}$ & $\begin{array}{l}\text { ENCONTRARON } \\
\text { NATERIAL }\end{array}$ & $\%$ \\
\hline UNA VEZ AL MES & $\begin{array}{l}59 \\
(9.2)\end{array}$ & 42 & 71.2 & & 17 & 28.8 \\
\hline SEMANALMENTE & $\begin{array}{l}158 \\
(24.6)\end{array}$ & 111 & $\overline{7} 0.3$ & & $4 \overline{7}$ & 29.7 \\
\hline $\begin{array}{l}\text { MAS DE UNA VEZ A LA } \\
\text { SEMANA }\end{array}$ & $\begin{array}{l}426 \\
(66.2)\end{array}$ & 291 & 68.3 & & 135 & 31,7 \\
\hline$T \circ T A \mathbb{L}:$ & $\begin{array}{c}642 \\
(100)\end{array}$ & 444 & 69.1 & & 199 & 30.9 \\
\hline
\end{tabular}


Cuadro 9

Comparación entre el semestre que cursaban los alumnos y el resultado que obtuvieron aquellos que acudieron a la biblioteca a consultar uno o varios materiales

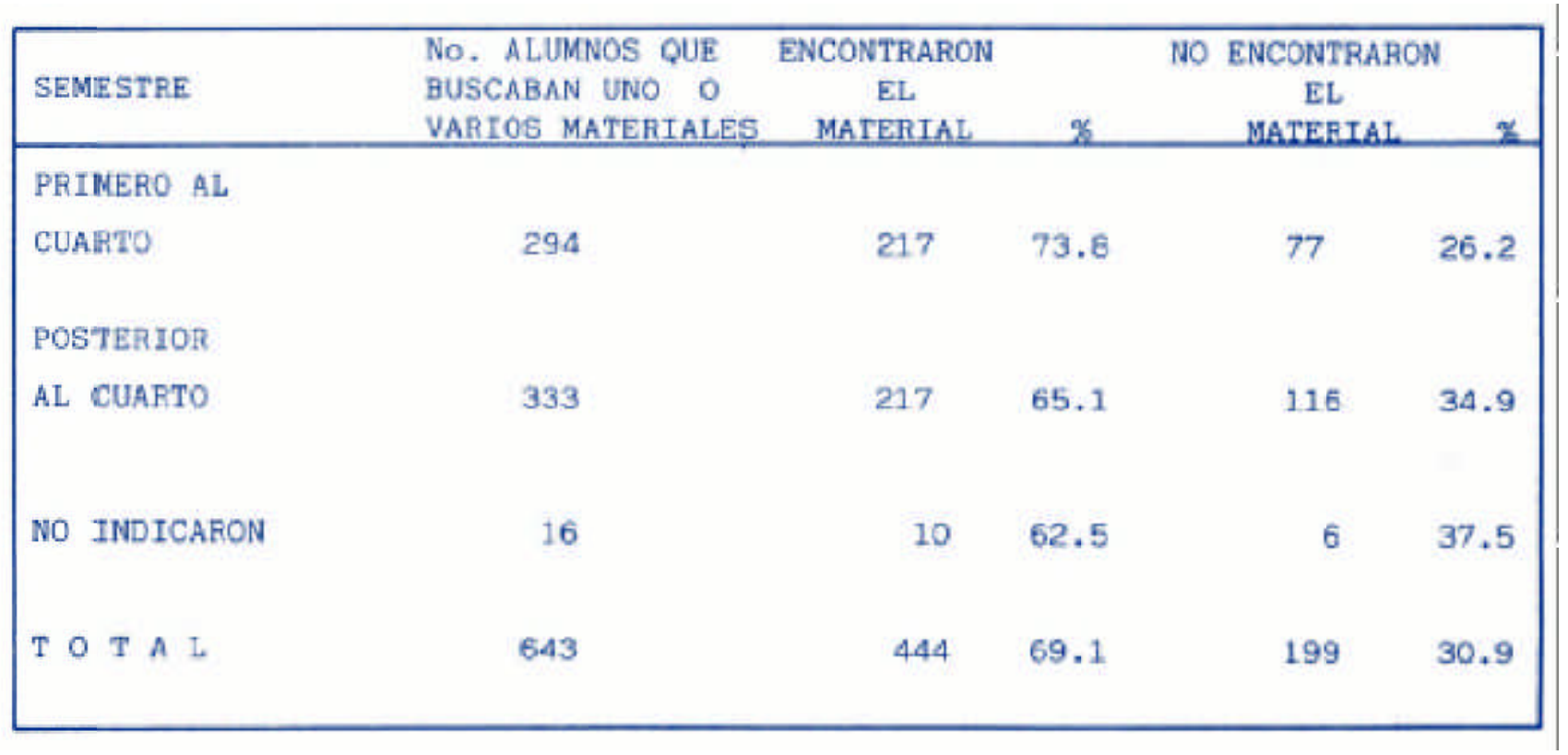

Cuadro 10

Influencia de la instrucción del uso del catálogo sobre la localización dentro de la biblioteca del material buscado

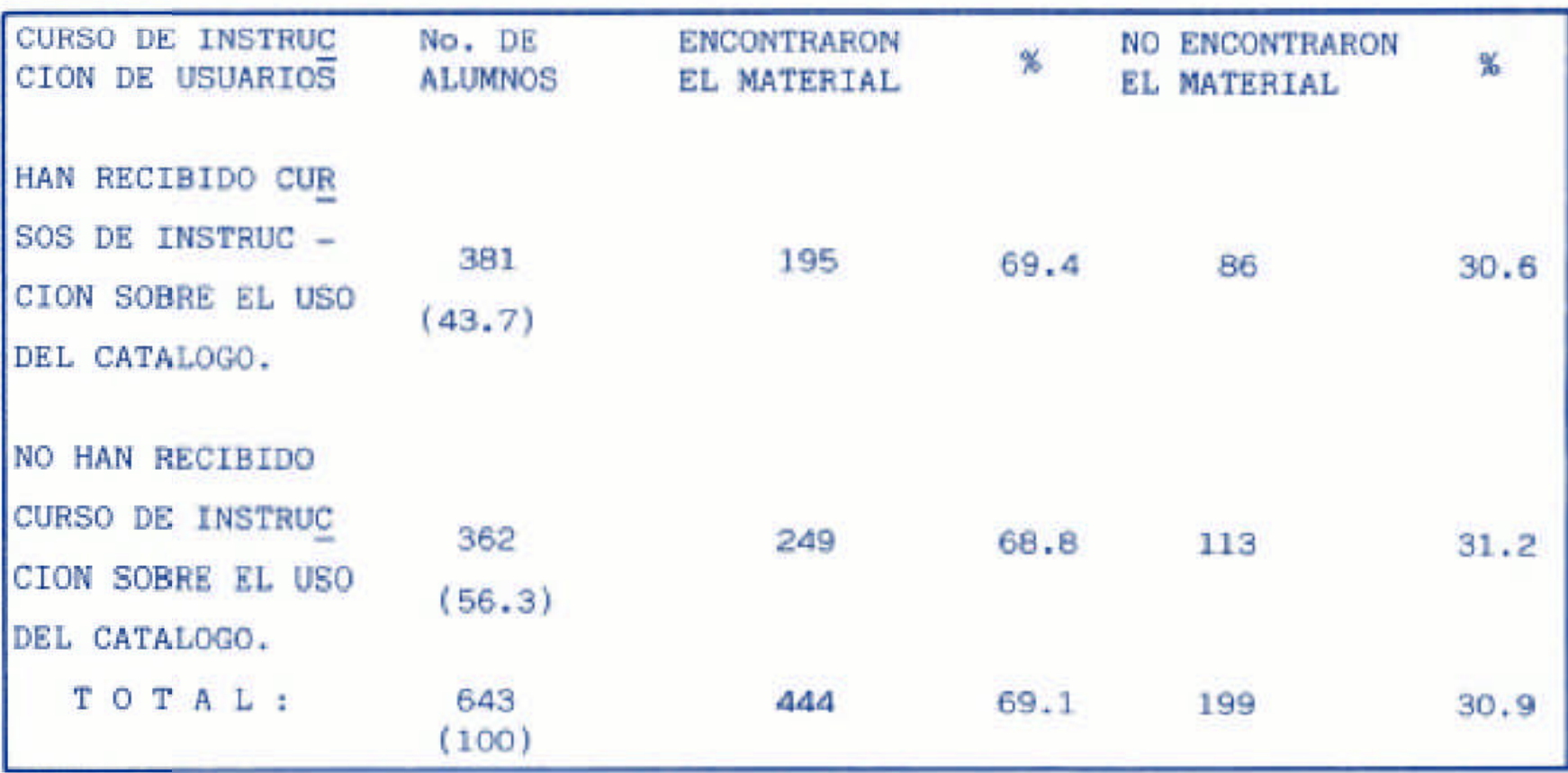


Cuadro 11

Relación entre el semestre que cursaban los alumnos y los datos sobre si habían o no recibido instrucción para el uso del catálogo

\begin{tabular}{|c|c|c|c|c|c|}
\hline SEMESTRE & No. $D E$ & $\begin{array}{l}\text { HAN RECIBIDO INS } \\
\text { TRUCCIOM (USO } \\
\text { DEL CATALOGO) }\end{array}$ & $\%$ & $\begin{array}{l}\text { INO IIAN RECIBIDO } \\
\text { INSTRUCCION } \\
\text { (USO DE CATALOGO) }\end{array}$ & $x$ \\
\hline $\begin{array}{l}\text { PRIMERO AL } \\
\text { CUARTO }\end{array}$ & 294 & 131 & 44.5 & 163 & 55.5 \\
\hline $\begin{array}{l}\text { POSTERIOR } \\
\text { AL CUAFTO }\end{array}$ & 333 & 144 & 43.2 & 189 & 56.8 \\
\hline NO INDICARON & 16 & 6 & 37.5 & 10 & 62.5 \\
\hline $\mathrm{T} O \mathrm{~T} A \mathrm{~L}$ & 643 & 281 & 43.7 & 362 & 56.3 \\
\hline
\end{tabular}

Cuadro 12

Relación entre los datos sobre los alumnos que recibieron instrucción sobre el uso del catálogo y la facilidad de su manejo

RECIBIERON INSTRUC CION SOBFE EL USO

DEL CATALOGO

\section{No. $D E$}

ALUMNOS

281

362

643
EL USO DEL CFT' LOGO LE PARECE FACIL
TE. USQ LEL CA. TALOGO LE PA= RECE DIFICIL

SI

241

85.8

40

14.2

No

265

73.2

97

26.8

T O T A :

506

78.7

137

21.3 
Cuadro 13

Relación entre el medio por el cual se recibió la instrucción del uso del catálogo y si considera a esta suficiente o insuficiente

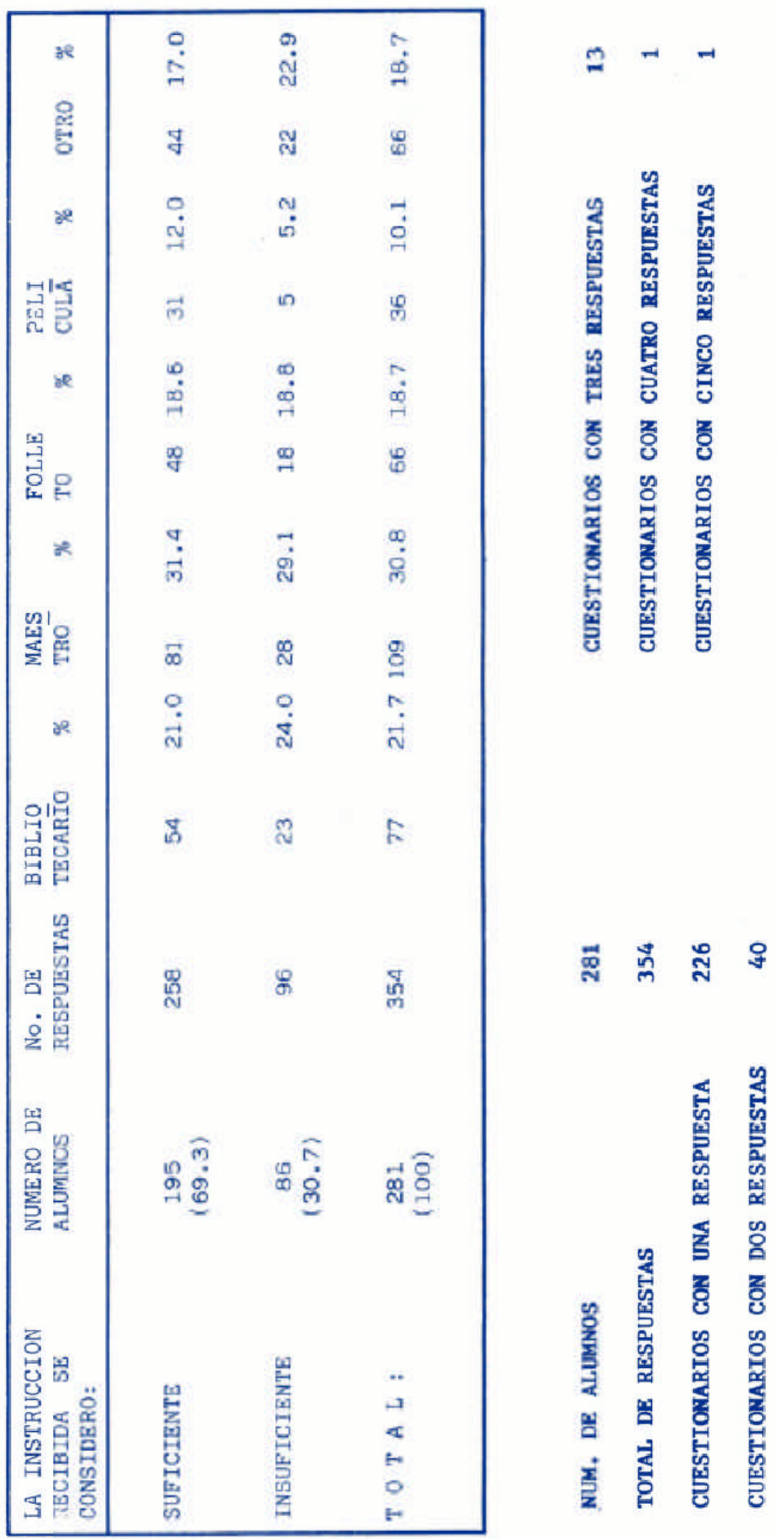




\section{E N C U E S T A}

Los alum nos de la Li cen cia tu ra en Biblio te co lo gía de la UNAM, es ta mos rea li zan dounaen cues ta so bre los ca tálogos de las bibliotecas. Los resultados que ésta arroje nos ayu da rán a conocer me jortusnecesidades, y en nuestro futuro ejercicio profesional a elaborar catálogos más efectivos, por lo cual agradecemos tu cooperación.

1. Datos Generales.

Carrera

Semestre

2. Frecuencia con que asistes a la biblioteca

Una vez al mes ( )

Semanalmente ( )

Más de una vez a la semana ( )

3. El objetivo principal de tu visita a la biblioteca fue:

$\begin{array}{ll}\text { Consultar una obra en particular } & \text { ( ) } \\ \text { Consultar varias obras sobre un tema } & \text { ( ) } \\ \text { Otras razones } & \text { ( ) }\end{array}$

4. En el caso de que el objetivo principal haya sido consultar una obra en particular anota los datos que conozcas. Autor

Título

5. En el caso de que el objetivo principal haya sido consultar varias obras sobre un tema anota el tema deseado.

6. Encontraste en la biblioteca el material que buscabas.
SI
( )
NO
( )

7. Para tratar de localizar las obras que necesitas:

Buscas en el catálogo de la biblioteca ( )

Preguntas al bibliotecario

( )

Buscas en la estantería

( )

Usas otro mecanismo

( )

8. Cuándo utilizas el catálogo buscas:
Un autor
( )
Un título
( )
Un tema
( )

9. Cuándo usas el catálogo encuentras que son:

Fáciles de entender y usar ( )

Difíciles de entender y usar ( )

No podría contestar

( )

10. Has recibido instrucciones de cómo usar el catálogo de esta o de otras bibliotecas.
SI
( )
$\mathrm{NO}$
( )

11. En caso afirmativo la explicación fue proporcionada por:
Un bibliotecario
( )
Un maestro
( )
Un folleto
( )
Una película o audiovisual
( )
Otros medios
( )

12. Consideras que la instrucción recibida para utilizar el catálogo de la biblioteca fue:
Suficiente
( )
Insuficiente
( ) 